\title{
Characterisation of fowl adenovirus (FAdV-8b) strain concerning the geographic analysis and pathological lesions associated with inclusion body hepatitis in broiler flocks in Turkey
}

\author{
Utku Yusuf Cizmecigil $^{1}$, Sajid Umar ${ }^{1,4}$, Aysun Yilmaz ${ }^{1}$, Erhan Bayraktar ${ }^{2}$, \\ Nuri Turan ${ }^{1}$, Besim Tali ${ }^{1}$, Ozge Aydin ${ }^{1}$, Hasan Emre Tali ${ }^{1}$, \\ Mehmetcan Yaramanoglu ${ }^{1}$, Semaha Gul Yilmaz ${ }^{1}$, Ahmet Kolukisa ${ }^{2}$, Jean-Remy Sadeyen ${ }^{3}$, \\ Munir Iqbal ${ }^{3}$, Huseyin Yilmaz ${ }^{1 凶}$ \\ ${ }^{1}$ Department of Virology, Veterinary Faculty, Istanbul University-Cerrahpasa, Avcilar, 34320 Istanbul, Turkey \\ ${ }^{2}$ CEVA Animal Health, Poultry Division, Büyükdere34453 Maslak, Istanbul, Turkey \\ ${ }^{3}$ Pirbright Institute, Pirbright Woking GU24 0NF, UK \\ ${ }^{4}$ Department of Veterinary Pathology, PMAS Arid Agriculture University, 46000 Rawalpindi, Pakistan \\ hyilmaz@istanbul.edu.tr
}

Received: July 10, 2019

Accepted: April 20, 2020

\begin{abstract}
Introduction: Fowl adenovirus can cause important diseases in chickens such as inclusion body hepatitis, hepatitis hydropericardium syndrome, and gizzard erosion and ulceration. Inclusion body hepatitis has been regularly reported from many countries. This is the first case report from Turkey, describing an outbreak of inclusion body hepatitis in broiler farms due to fowl adenovirus- 8 b (FAdV-8b). Material and Methods: Broiler flocks with mortality about $10 \%$ were visited in Turkey, and necropsy was performed on dead birds. Samples were subjected to PCR assay to detect FAdV and other viral pathogens. After sequencing, phylogenetic analysis was performed and the nucleotide sequences of hexon genes were compared with the FAdV sequences data available in GenBank. Results: Clinical signs such as anorexia, depression, ruffled feathers, huddling, and greenish diarrhoea were observed. Mortality started at the $8^{\text {th }}$ day of age and ranged from $10 \%$ to $14 \%$. Necropsy showed severe hepatitis, jaundice, and pancreatitis. The main necropsy findings included a pale, enlarged, haemorrhagic, and friable liver along with swollen and haemorrhagic kidneys and spleen. PCR and sequence analysis revealed the presence of fowl adenovirus serotype 8b (FAdV-E). Conclusion: This is the first report on characterisation and the pathological lesions associated with FAdV in broilers in Turkey. Our findings suggest that FAdV strains could be an emerging pathogen in Turkish broilers and could actively contribute to hepatitis and immunosuppression.
\end{abstract}

Keywords: broilers, fowl adenovirus, inclusion body hepatitis, genotyping, Turkey.

\section{Introduction}

Fowl adenoviruses (FAdVs) cause a variety of diseases in chickens such as inclusion body hepatitis (IBH), hydropericardium hepatitis syndrome (HHS), and adenoviral gizzard erosion (AGE), and they lead to economic losses all over the world $(11,22,31)$. FAdVs are non-enveloped double-stranded DNA viruses, which belong to the Adenoviridae family $(6,36)$. FAdVs are transmitted vertically and horizontally, but the highest titres are found in faeces (4). FAdVs are classified into five different species (FAdV-A to FAdV-E) due to their molecular structure, and also into 12 serotypes (FAdV-1 to $-8 \mathrm{a}$ and $8 \mathrm{~b}$ to -11 ), as a result of cross-neutralisation tests and melting curve analysis (6). At least 12 genotypes were identified within the five FAdV species based on the hexon gene sequences (11, 33, 34). Recent reports have documented that FAdVs involved in IBH are mainly related to species $\mathrm{D}$ (FAdV-2 and FAdV-11) and E (FAdV-8a and 
FAdV-8b) (31). FAdV strains responsible for HHS identify as species $\mathrm{C}$ and belong to FAdV-4. They are highly pathogenic to chickens. Species A strain FAdV-1 has been isolated from most cases of gizzard erosion and ulceration (4).

Hexon, fibre, and penton are main structural proteins of the FAdVs capsid. The hexon gene is vulnerable to mutations and is used for serotyping as it harbours the major neutralising epitope. PCR using different primer sets, which can amplify the various regions of the hexon gene, followed by restriction enzyme digestion or nucleotide sequencing of the products, allows the differentiation of field isolates to species and serotypes (13). The combination of PCR and high-resolution melting curve analysis has also provided an accurate and rapid genotyping technique for the identification of FAdV serotypes (33). Inactivated and live vaccines are used in some countries where FAdVs are endemic and outbreaks of the disease are frequent $(30,31)$. However, commercial vaccines are not used against FAdVs in Turkey due to lack of knowledge about the disease distribution and circulating viruses.

Although IBH has been reported from clinical outbreaks in many countries, there is still a lack of information about the occurrence of this disease complex in Turkey. To the best of our knowledge, this is the first report on an IBH outbreak in Turkish broiler flocks.

\section{Material and Methods}

Farms and collection of samples. Two commercial broiler flocks (Ross 308) with sudden onset of mortality of about $10 \%$ were visited in Aegean region of Turkey in spring 2019. Each farm consisted of two houses and each house had a capacity of 25,000 broiler chickens. The birds were reared in a controlled housing system. In these units, the production cycle lasted about $42-45$ days. The houses were routinely disinfected between rearing periods, and transport tracks were also disinfected before each transfer. All entrances were kept under control for biosecurity, catered for with a disinfection facility in door entrances. Clothes and boots were changed at the entrance, and crumble-type feed was used. Wood shavings were used as litter material. According to information from the farmers, chickens on neither of the farms visited were vaccinated against infectious bursal disease virus (IBDV), avian metapneumovirus (aMPV), or infectious laryngotracheitis virus (ILTV). However, birds were vaccinated with live NDV and IBV vaccines via spray as well as inactivated NDV by the subcutaneous route in the hatchery.

No clinical cases of such infection had been observed at those production sites or broiler integrations in the past. The cases investigated in this study were seen in one broiler house of each flock of which about 2,500 birds showed clinical signs. Young (eight days old) broiler chicks were usually found dead but were occasionally seen in an extremely depressed condition shortly before death. Death occurred within a few hours of the initial observation of disease signs. Necropsy was performed on 30 chicks which had died a short time before. Samples of the liver and spleen were taken into sterile cups and brought under temperature-controlled conditions to the Department of Virology of the Veterinary Faculty of Istanbul University-Cerrahpasa.

Nucleic acid extraction and reverse transcription. Tissue samples were ground and homogenised in phosphate-buffered saline (PBS; $0.1 \mathrm{M}, \mathrm{pH}$ 7.2). Viral DNA was extracted from tissue homogenates to detect FAdVs using a PureLink genomic DNA Mini Kit (Invitrogen, Carlsbad, CA, USA). To detect other viruses, RNA was extracted from samples with the use of Pure Link RNA mini kits (Invitrogen) according to the manufacturer's instructions. RNA was eluted in $30 \mu \mathrm{L}$ of elution buffer and stored at $-20^{\circ} \mathrm{C}$ until further use. Reverse transcription was performed with a high-capacity cDNA Reverse Transcription Kit (Applied Biosystems, Waltham, MA, USA) following the manufacturer's protocol. Briefly, a total of $10 \mu \mathrm{L}$ of RNA was used in $20 \mu \mathrm{L}$ of RT reaction mixture. The RT was performed at $25^{\circ} \mathrm{C}$ for $10 \mathrm{~min}$, followed by $37^{\circ} \mathrm{C}$ for $2 \mathrm{~h}$, and finally at $85^{\circ} \mathrm{C}$ for $5 \mathrm{~min}$.

PCR for amplification of the hex loop-1 gene of fowl adenovirus. A 590-bp region of the hexon gene was amplified by PCR to confirm the presence of viral DNA and for sequencing. One set of primers binding to the hex loop 1 (L1) gene was used to amplify L1. The primers were as follows: Hex L1-F 5'-ATGGGAGCSACCTAYTTCGACAT-3' (301-323) as the forward primer and Hex L1-R 5'-AAATTGTCCCKRAANCCGATGTA-3' (890-868) as the reverse primer $(25,30)$. The optimised PCR reaction consisted of a total volume of $25 \mu \mathrm{L}$ of reaction mixture containing $2 \mu \mathrm{L}(10 \mu \mathrm{M})$ of each forward and reverse primer, $12.5 \mu \mathrm{L}$ of Maxima Hot Start PCR Master Mix (Thermo Scientific, Waltham, MA, USA), $4.5 \mu \mathrm{L}$ of nuclease-free water, $2 \mu \mathrm{L}$ of $\mathrm{MgCl}_{2}$, and $2 \mu \mathrm{L}$ of DNA. Amplification was carried out in a Chromo4 thermal cycler (Bio-Rad, Hercules, CA, USA) under the protocol described previously (30). The reaction conditions were $10 \mathrm{~min}$ at $95^{\circ} \mathrm{C}$ for initial denaturation followed by 40 cycles of denaturation at $95^{\circ} \mathrm{C}$ for $15 \mathrm{~s}$, annealing at $56^{\circ} \mathrm{C}$ for $30 \mathrm{~s}$, and extension at $72^{\circ} \mathrm{C}$ for $45 \mathrm{~s}$, and a final extension at $72^{\circ} \mathrm{C}$ for $5 \mathrm{~min}$. For all PCR reactions, positive and negative controls were included. A known positive field sample was used as the positive control, while nuclease-free water was included as the negative control in place of the DNA template. PCR products were analysed in Ultrapure $1.5 \%$ agarose gel (Invitrogen, Merelbeke, Belgium), containing SYBR Safe DNA gel nucleic acid stain (Thermo Fisher 
Scientific, Tracy, CA) using $1 \times$ TBE electrophoresis running buffer. Samples were also screened by PCR for the presence of infectious bronchitis virus, infectious bursal disease virus, avian metapneumovirus, and infectious laryngotracheitis virus as part of routine diagnostic work.

Sequencing and phylogenetic analysis. Amplified PCR products from the liver samples belonging to two broiler flocks were sent for sequencing to a commercial company (MedSanTek, Turkey). Raw nucleotide sequences of the hexon genes of FAdV were edited, aligned, and used for phylogenetic analysis. The nucleotide sequences of the hexon genes were compared with the FAdV sequences data available in the National Centre for Biotechnology Information, and the phylogenetic relationship was established. All of the sequences were aligned using the CLUSTAL-W tool with MEGA7 software (9). Distance-based neighbour-joining trees were constructed by using the P-distance model (29). Turkish FAdV field strains were identical, and thus only one representative sequence was submitted to GenBank under the submission number MN052902.

\section{Results}

Clinical findings. The sudden onset of deaths, depression, huddling with ruffled feathers, lethargy, reduced feed intake, reduced weight gain, and lack of uniformity of the flock were prominent. Greenish diarrhoea was seen from some chicks (Fig. 1A). The mortality rate ranged from $10 \%$ to $14 \%$ and started at eight days of age in affected broiler flocks. The mortality peaked on the $16^{\text {th }}$ day and gradually declined to normal by the $24^{\text {th }}$.

Necropsy. At necropsy, birds showed severe hepatitis and jaundice. The livers were friable, enlarged, and pale yellow with multiple petechial haemorrhages and small white foci (Fig. 1B). In sudden death cases, haematoma was also observed on some livers (Fig. 1C). The kidneys were swollen and haemorrhagic (Fig. 1D). The spleens were slightly enlarged and had necrotic spots on their surface (Fig. 1E). Moreover, the pancreas was pale and swollen (Fig. 1F).

PCR analysis, sequencing, and phylogeny. Amplification of the hexon gene fragment resulted in a 590 bp PCR product (Fig. 2). Results from the nucleotide BLAST for the sequences revealed $96 \%$ $99.48 \%$ similarity with the genomic sequence of the FAdV isolates present in the BLAST database (BLAST, NCBI http://blast.ncbi.nlm.nih.gov/Blast.cgi.). Nucleotide sequences from the liver matched FAdV species E isolates of serotype FAdV 8b.
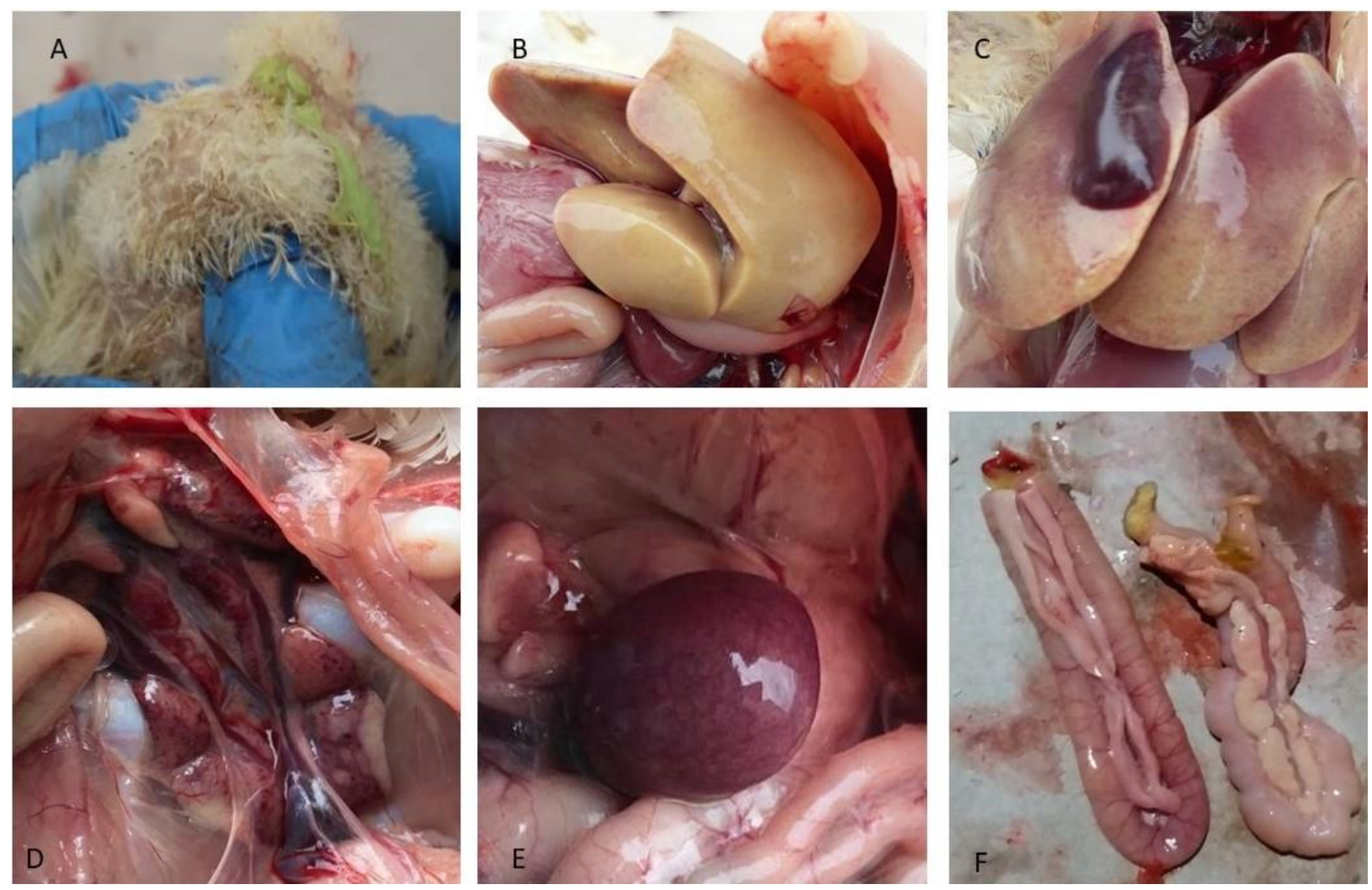

Fig. 1. Gross lesions in eight-day-old broiler chicks. A - greenish diarrhoea; B and C - friable, enlarged, and pale yellow liver showing haematoma, miliary necrosis, and multiple petechial haemorrhages; D - swollen and haemorrhagic kidneys; E - enlarged spleen with necrotic spots; $\mathrm{F}$ - pale and swollen pancreases 
The sequencing analysis of the partial hexon gene revealed the high similarity of the FAdV-E (FAdV-8b) strain characterised in this study to those from Indonesia, Canada, Peru, and China (MK692992, EF685489, KX755572, and KU981139), and its difference from Egyptian, Iranian, Lebanese, Pakistani, Austrian, Italian, Brazilian, and Australian strains reported to GenBank (Fig. 3).

In this study, samples were also screened for the presence of possible mixed viral infections as part of routine diagnostic work using RT-PCR. However, none of the samples were found to be positive for the tested field viruses.

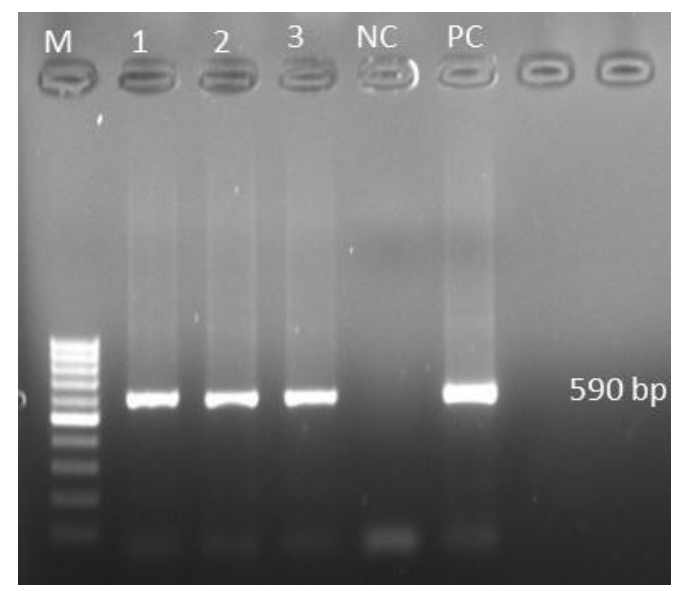

Fig. 2. Molecular identification of inclusion body hepatitis virus (FAdV) by PCR. M - 100 bp molecular marker;, 1, 2, 3 - liver samples;, NC - negative control;, PC - positive control

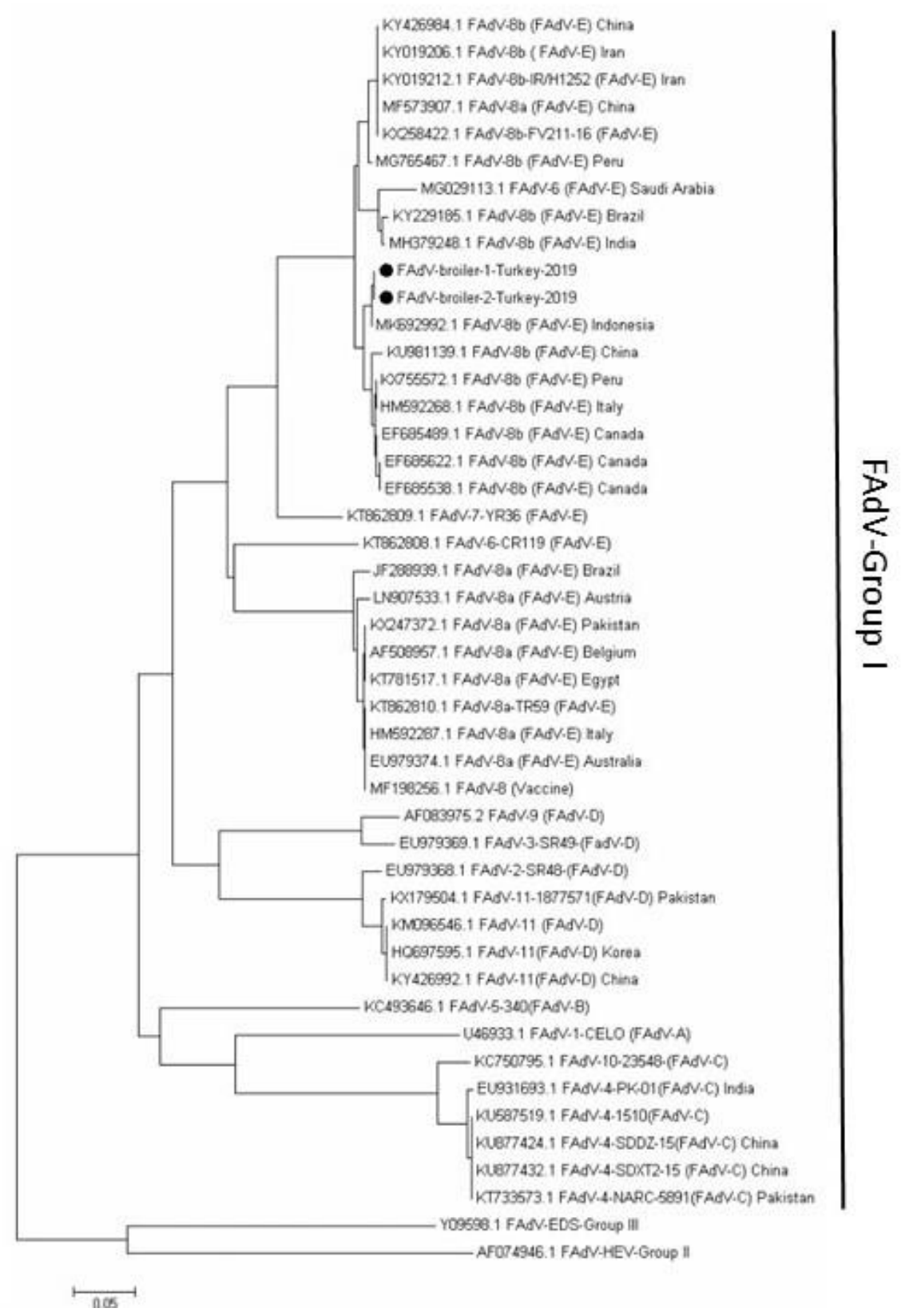

Fig. 3. The phylogenetic relationship of hexon gene sequences of FAdV based on the neighbourjoining method. Turkish strains obtained in the present study have been highlighted with black circles 


\section{Discussion}

Turkey has one of the largest poultry farming industries in the world. Intensive commercial farming and insufficient control measures have led to the emergence of new viral infections. Thus, epidemiologic research is very important for monitoring disease outbreaks and developing vaccines. FAdV infections have become a matter of great concern for poultry farmers worldwide (31). IBH not only causes mortality but can also lead to a drop in production, resulting in severe economic losses. Historically, IBH was first described in US American domestic chickens in 1963 and then rapidly spread over the world $(5,34)$. A higher number of clinical cases of FAdV infection have been reported in recent years, and multiple FAdV strains have been isolated from sick animals in many countries $(8,14,22,23,31)$. Unfortunately, there is no conclusive evidence or published data regarding FAdV in Turkish chickens. This reinforces the necessity of promoting molecular surveys on this emerging pathogen in broilers and the need to study its actual role in clinically overt situations. This study describes an outbreak of IBH in Turkish broiler flocks.

In this study, high acute mortality (10\%-14\%) started at 8 days of age in affected broiler flocks. The mortality peaked on the $16^{\text {th }}$ day and gradually declined to be normal again at 24 days of age. Although IBH can affect broilers of all ages, young chicks are found to be more susceptible during the first two weeks even when in immunologically intact. There is a clear age effect with avian adenoviruses, as the age of the host increases, the degree of multiplication of the viruses within the host is restricted and the mortality decreases (27). There was no accumulation of straw coloured fluid in the pericardial sac, indicating that there was no relation with HHS. This is most likely to be because of the different viral strains and serotypes of FAdV causing infection in this study, which is an explanation with precedents from other authors $(14,23,26,31)$. Mortality during IBH outbreaks is normally between $2 \%$ and $10 \%$ of the flock, but up to $30 \%$ has been described in case of co-infection with other immunosuppressive causative agents $(3,31)$. As reported previously $(1,17,23,27,31)$, typical necropsy observations such as an enlarged and pale liver, enlarged and haemorrhagic kidneys and spleen, and clinical findings were observed in the IBH cases of the present study. In addition, a pale and enlarged pancreas along with greenish diarrhoea were also observed in some chicks, as was also reported by others $(1,25)$.

In the present study, phylogenetic analysis of the isolates revealed circulation of FadV species $\mathrm{E}$ and serotype $8 \mathrm{~b}$ in Turkish broiler flocks. The sequences of FAdV-E obtained in this study shared $100 \%$ nucleotide identity with each other and between $96 \%$ and $99.48 \%$ identity with the previously published sequences from Indonesia, Canada, Peru, and China, suggesting potential virus transmission from these countries possibly via trades and primary breeders or breeder replacements. Turkish FAdV strains formed a different cluster with other FAdV strains reported so far in different countries of the world including Egypt (2019), Brazil (2018), Iran (2015), Italy (2007), Russia (1997), Pakistan (1989), and Australia (1986). FAdV-E has spread worldwide and been reported in Australia, Belgium, Canada, China, Egypt, France, Hungary, Italy, Malaysia, Mexico, New Zealand, Poland, and Spain and the USA $(26,31)$. IBH outbreaks have also been reported from countries neighbouring Turkey. Recently, FadV-D and FAdV-E were detected and characterised from IBH outbreaks in Egypt and Saudi Arabia (3, 26). FAdV-6 and FAdV-2 were detected from Saudi poultry flocks by Mohamed et al. (15). In Lebanon, FAdV-11 was found in commercial broiler flocks (32), and in Iran, two FAdV species D and E (FAdV-11, FAdV-8b) were linked with outbreaks of IBH $(7,16,18)$. Similarly, an investigation of the outbreaks of IBH in Australia, South Africa, Austria, and Spain reported FAdV-8b or FAdV-11 in broiler flocks $(10,24,30,34)$. Also, an epidemiological investigation of outbreaks of FAdV in commercial chickens in Korea indicated that all FAdVs types $8 \mathrm{~b}$ and 11 had relevance to IBH lesions (2). FAdVs associated with IBH outbreaks in Canada were genetically related to FAdV-2, FAdV-8b, and FAdV-11 (4, 23). In Japan, FAdV-D (12) and in Slovenia, FAdV-E serotype $8 \mathrm{~b}$ (35) were found to be the causative agent of IBH, and in Poland FAdV-7 caused IBH and was confirmed in broiler chickens (19).

Immunosuppressive agents, such as infectious bursal disease virus (IBDV), chicken infectious anaemia virus (CIAV), and Marek's disease virus (MDV) $(20,21)$ are predisposing factors to IBH outbreaks or can exacerbate clinical manifestations concurrently with FAdV infections (16). Studies in Canada, the United States, and New Zealand indicated that IBH occurred as a primary disease, without association with IBDV or CIAV (4, 23).

In conclusion, the current study is the first report on the molecular characterisation of FAdVs in Turkish broilers. Our findings suggest that FAdVs could be emerging pathogens in Turkish poultry flocks and could cause severe hepatitis in young chicks. Further studies have to be performed to assess the prevalence and pathogenicity of serotypes of fowl adenoviruses and their association with other factors in poultry flocks in Turkey. Identification of FAdV serotypes is important in epidemiological studies of the disease outbreaks, the development of preventative measures, and the adoption of vaccination strategies.

Conflict of Interests Statement: The authors declare that there is no conflict of interests regarding the publication of this article. 
Financial Disclosure Statement: The samples analysed in this study were from a project funded by the Istanbul University-Cerrahpasa (BAP-Project no: 27243).

\section{Animal Rights Statement: Not applicable.}

Acknowledgements: We would like to thank Istanbul University-Cerrahpasa for funding this study.

\section{References}

1. Ahamad D.B., Selvaraj J., Sasikala M., BabuPrasath N.: Inclusion body hepatitis in chicken. Indian Vet J 2016, 93, 34-35.

2. Choi K.S., Kye S.J., Kim J.Y., Jeon W.J., Lee E.K., Park K.Y., Sung H.W.: Epidemiological investigation of outbreaks of fowl adenovirus infection in commercial chickens in Korea. Poult Sci 2012, 91, 2502-2506.

3. El-Tholoth M., Abou El-Azm K.I.: Molecular detection and characterization of fowl adenovirus associated with inclusion body hepatitis from broiler chickens in Egypt. Trop Anim Health Prod 2019, 51, 1065-1071.

4. Gomis S., Goodhope A.R., Ojkic A.D., Willson P.: Inclusion body hepatitis as a primary disease in broilers in Saskatchewan, Canada. Avian Dis 2006, 50, 550-555.

5. Helmboldt C.F., Frazier M.N.: Avian hepatic inclusion bodies of unknown significance. Avian Dis 1963, 7, 446-450.

6. Hess M.: Detection and differentiation of avian adenoviruses: a review. Avian Pathol 2000, 29, 195-206.

7. Hosseini H., Morshed R.: Molecular identification of fowl adenovirus associated with inclusion body hepatitis in Iran. Iran J Virol 2012, 6, 7-12.

8. Kaján G.L., Kecskeméti S., Harrach B., Benkő M.: Molecular typing of fowl adenoviruses, isolated in Hungary recently, reveals high diversity. Vet Microbiol 2013, 167, 357-363.

9. Kumar S., Stecher G., Tamura K.: MEGA7: molecular evolutionary genetics analysis, version 7.0 for bigger datasets. Mol Biol Evol 2016, 33, 1870-1874.

10. Maartens L.H., Joubert H.W., Aitchison H., Venter E.H.: Inclusion body hepatitis associated with an outbreak of fowl adenovirus type 2 and type $8 \mathrm{~b}$ in broiler flocks in South Africa. J S Afr Vet Assoc 2014, 85, 1-5.

11. Marek A., Günes A., Schulz E., Hess M.: Classification of fowl adenoviruses by use of phylogenetic analysis and high-resolution melting-curve analysis of the hexon L1 gene region. J Virol Methods 2010, 170, 147-154.

12. Mase M., Nakamura K., Minami F.: Fowl adenoviruses isolated from chickens with inclusion body hepatitis in Japan, 20092010. J Vet Med Sci 2012, 74, 1087-1089.

13. Meulemans G., Boschmans M., Berg T.P., Decaesstecker M.: Polymerase chain reaction combined with restriction enzyme analysis for detection and differentiation of fowl adenoviruses. Avian Pathol 2001, 30, 655-660.

14. Mittal D., Jindal N., Tiwari A.K., Khokhar R.S.: Characterization of fowl adenoviruses associated with hydropericardium syndrome and inclusion body hepatitis in broiler chickens. Virus Disease 2014, 25, 114-119.

15. Mohamed M.H.A., El-Sabagh I.M., Abdelaziz A.M., Al-Ali A.M., Alramadan M., Lebdah M.A., Al-Ankari A.R.S.: Molecular characterization of fowl aviadenoviruses species D and $\mathrm{E}$ associated with inclusion body hepatitis in chickens and falcons indicates possible cross-species transmission. Avian Pathol 2018, 47, 384-390.

16. Morshed R., Hosseini H., Langeroudi A. G., Fard M.H.B., Charkhkar S.: Fowl adenoviruses D and E cause inclusion body hepatitis outbreaks in broiler and broiler breeder pullet flocks. Avian Dis 2017, 61, 205-210.

17. Nakamura K., Mase M., Yamamoto Y., Takizawa K., Kabeya M., Wakuda T., Matsuda M., Chikuba T., Yamamoto Y., Ohyama T., Takahashi K., Sato N., Akiyama N., Honma H., Imai K.: Inclusion body hepatitis caused by fowl adenovirus in broiler chickens in Japan, 2009-2010. Avian Dis 2011, 55, 719-723.

18. Nateghi E., Razmyar J., Bassami M.R.: Molecular characterization of avian adenoviruses in Iranian broiler flocks. Iran J Vet Res 2014, 15, 164-167.

19. Niczyporuk J.S.: Molecular characterisation of fowl adenovirus type 7 isolated from poultry associated with inclusion body hepatitis in Poland. Arch Virol 2017, 162, 1325-1333.

20. Niczyporuk J.S., Samorek-Salamonowicz E., Czekaj H.: Occurrence of adenovirus field strains in birds infected with Marek's disease virus. Bull Vet Inst Pulawy 2012, 56, 435-440.

21. Niczyporuk J.S., Woźniakowski G., Samorek-Salamonowicz E., Czekaj H.: Effect of fowl adenovirus (FAdV-7) infection on the replication of turkey herpesvirus FC126 in chicken embryo fibroblast cultures. Bull Vet Inst Pulawy 2012, 56, 441-446.

22. Niu Y., Sun Q., Zhang G., Sun W., Liu X., Xiao Y., Liu S.: Epidemiological investigation of outbreaks of fowl adenovirus infections in commercial chickens in China. Transbound Emerg Dis 2018, 65, 121-126.

23. Ojkic D., Martin E., Swinton J., Vaillancourt J.P., Boulianne M., Gomis S.: Genotyping of Canadian isolates of fowl adenoviruses. Avian Pathol 2008, 37, 95-100.

24. Oliver-Ferrando S., Dolz R., Calderón C., Valle R., Rivas R., Pérez M., Majó N.: Epidemiological and pathological investigation of fowl aviadenovirus serotypes $8 \mathrm{~b}$ and 11 isolated from chickens with inclusion body hepatitis in Spain (20112013). Avian Pathol 2017, 46, 157-165.

25. Pilkington P., Brown T., Villegas P., McMurray B., Page R.K., Rowland G.N., Thayer S.G.: Adenovirus-induced inclusion body hepatitis in four-day-old broiler breeders. Avian Dis 1997, 41, 472-474.

26. Radwan M.M., El-Deeb A.H., Mousa M.R., El-Sanousi A.A., Shalaby M.A.: First report of fowl adenovirus 8a from commercial broiler chickens in Egypt: molecular characterization and pathogenicity. Poult Sci 2019, 98, 97-104.

27. Rahimi M., Minoosh Siavosh Haghighi Z.: Adenovirus-like inclusion body hepatitis in a flock of broiler chickens in Kermanshah province, Iran. Vet Res Forum 2015, 6, 95-98.

28. Raue R., Gerlach H., Müller H.: Phylogenetic analysis of the hexon loop 1 region of an adenovirus from psittacine birds supports the existence of a new psittacine adenovirus (PsAdV). Arch Virol 2005, 150, 1933-1943.

29. Saitou N., Nei M.: The neighbor-joining method: a new method for reconstructing phylogenetic trees. Mol Biol Evol 1987, 4, 406-425.

30. Schachner A., Marek A., Grafl B., Hess M.: Detailed molecular analyses of the hexon loop-1 and fibers of fowl aviadenoviruses reveal new insights into the antigenic relationship and confirm that specific genotypes are involved in field outbreaks of inclusion body hepatitis. Vet Microbiol 2016, 186, 13-20.

31. Schachner A., Matos M., Grafl B., Hess M.: Fowl adenovirusinduced diseases and strategies for their control - a review on the current global situation. Avian Pathol 2018, 47, 111-126.

32. Shaib H., Ramadan N., Mahmoud G., Nassif G., Chedid S.: Outbreak of inclusion body hepatitis-causing adenovirus in Lebanese broiler flocks. EC Microbiol 2017, 13, 92-101.

33. Steer P.A., Kirkpatrick N.C., O'Rourke D., Noormohammadi A.H.: Classification of fowl adenovirus serotypes by use of highresolution melting-curve analysis of the hexon gene region. J Clin Microbiol 2009, 47, 311-321.

34. Steer P.A., O’Rourke D., Ghorashi S.A., Noormohammadi A.H.: Application of high-resolution melting curve analysis for typing of fowl adenoviruses in field cases of inclusion body hepatitis. Aust Vet J 2011, 89, 184-192. 
35. Zadravec M., Slavec B., Krapez U., Kajan G.L., Racnik J., Juntes P., Jursic C.R., Benko M., Zorman R.O.: Inclusion body hepatitis (IBH) outbreak associated with fowl adenovirus type $8 \mathrm{~b}$ in broilers. Acta Vet (Beogr) 2013, 63, 101-110.
36. Zhao J., Zhong Q., Zhao Y., Hu Y., Zhang G.: Pathogenicity and complete genome characterization of fowl adenoviruses isolated from chickens associated with inclusion body hepatitis and hydropericardium syndrome in China. PLoS One 2015, 10, 0133073 